\title{
Percepção visual no Tremor Essencial: Uma Revisão Sistemática*
}

\author{
Thiago Monteiro Paiva Fernandes ${ }^{1, * *}\left(\mathbb{D}\right.$, Debora da Silva Firino Felismino ${ }^{1}$, \\ Natalia Leandro de Almeida ${ }^{1}$ (D) \& Natanael Antonio dos Santos ${ }^{1}$ (D) \\ ${ }^{1}$ Universidade Federal da Paraíba (UFPB), João Pessoa, PB, Brasil
}

\begin{abstract}
RESUMO - Investigou-se a relação entre percepção visual e tremor essencial (TE). Realizou-se uma revisão dos estudos publicados sobre o tema nas bases de dados PubMed, BIREME, CINAHL, Web of Science, PsycINFO, SciELO, SCOPUS e ELSEVIER desde a origem até 05 de janeiro de 2017. A triagem, extração de dados e avaliação foram realizadas por dois revisores. Para avaliar a qualidade dos estudos utilizou-se as diretrizes do National Institutes of Health (NIH). De 132 estudos potencialmente elegíveis, 12 foram selecionados de acordo com os critérios de elegibilidade. Os resultados indicaram que pessoas com TE apresentam baixa sensibilidade visual quando comparados com indivíduos saudáveis, apesar de não apresentarem prejuízos na visão de cores. Apesar da heterogeneidade dos dados, observamos que existem prejuízos visuais em pessoas com TE e estes podem ser uma das principais características clínicas não-motoras.
\end{abstract}

PALAVRAS-CHAVE: tremor essencial, tremor, percepção visual, visão

\section{Visual Perception in Essential Tremor:A Systematic Review}

\begin{abstract}
It was investigated whether essential tremor (ET) patients would exhibit visual impairments. Studies published in the following databases were reviewed: PubMed, BIREME, CINAHL, Web of Science, PsycINFO, SciELO, SCOPUS, and ELSEVIER, from inception to January 05th, 2017. Two independent reviewers performed screening, data extraction, and assessment of the data. The NIH assessment tools used to assess study quality. Out of 132 references, 12 were selected according to the eligibility criteria. Results indicated that ET patients have lower visual sensitivity when compared to control groups. There was no significant evidence for color vision impairment. Despite the findings for heterogeneity, visual impairments in ET patients were observed, and these may be one of the main non-motor clinical characteristics.
\end{abstract}

KEYWORDS: tremor, essential tremor, visual perception, vision

O Tremor Essencial (TE) é comumente caracterizado como um tremor postural ou cinético, entre 4 e $12 \mathrm{~Hz}$ (Jiménez-Jiménez et al., 2010a), que afeta bilateralmente os braços, com ou sem manifestação quando o indivíduo está em ação. Em alguns casos, pode afetar também cabeça, queixo e voz, além de outros locais do corpo (Gitchel, Wetzel, \& Baron, 2013a; Jiménez-Jiménez et al., 2010a). O TE é o transtorno de movimento mais comum entre adultos, (Benito-León, 2014; Benito-León et al., 2015) e apesar de sua patogenia não ser completamente entendida (Benito-León, 2008, 2014), estima-se que aproximadamente $4 \%$ das pessoas acima de 40 anos sejam acometidas (Gitchel et al., 2013).
Além das alterações motoras já conhecidas, o TE pode ainda estar associado a outras manifestações não-motoras, como déficits cognitivos, demência, sintotologia depressiva (Benito-León et al., 2015) e alterações na percepção visual (Koller, Busenbark, \& Miner, 1994).

Estudos de neuroimagem traçaram paralelos importantes acerca da importância do envolvimento do cerebelo no transtorno (Deuschl, Wenzelburger, Löffler, Raethjen, \& Stolze, 2000; Filip, Lungu, Manto, \& Bareš, 2016). Deste modo, o TE é mediado pela associação entre a conexão cerebelo-tálamo-cortical (Benito-León et al., 2015; Marsden, Ashby, Limousin-Dowsey, Rothwell, \& Brown, 2000).

\footnotetext{
* Agradecimentos: Os autores gostariam de agradecer as contribuições de Rafaela Martins Rodrigues durante o processo de reformulação do presente trabalho.

** E-mail: thiagompfernandes@gmail.com

n Submetido: 16/01/2017; Revisado: 10/05/2018; Aceito: 28/05/2018.
} 
No que diz respeito a conexão tálamo-cortical que envolve o TE, destaca-se que a percepção visual, uma das portas de entrada da cognição (Cornsweet, 2012; Pylyshyn, 1999), também é mediada pela conexão (Gazzaniga, 2004). A informação visual decorrente da retina desencadeia reações que alcançam o núcleo geniculado lateral do tálamo e posteriormente o córtex visual primário, perpassando os caminhos subjacentes ao TE (Piñero, Monllor, Moncho, Camps, \& de Fez, 2015; Sharifi, Nederveen, Booij, \& van Rootselaar, 2014).

Durante o processo de neurulação, a neuroectoderma fomenta o desenvolvimento do sistema nervoso central e da retina. Assim, sendo uma extensão embrionária do encéfalo, a retina é uma das regiões mais estudadas do sistema nervoso central devido a sua posição anatômica (Adams \& Nasrallah, 2017; Schönfeldt-Lecuona et al., 2016). Neste sentido, a retina permite uma investigação não invasiva do processamento visual em termos ópticos e neurais (Schönfeldt-Lecuona et al., 2016). Com isto em mente, diversas medidas foram estabelecidas para caracterizar $o$ processamento visual, tal como a função de sensibilidade ao contraste, sensibilidade visual cromática (ou visão de cores), rastreamento do movimento ocular e perimetria.

Considerando os aspectos supracitados de comunalidade das vias de conexão, bem como a relativa facilidade de mensuração da via óptica, identificou-se a necessidade de realização de uma revisão que inclua publicações relevantes para promover uma visão geral da associação entre percepção visual e TE, buscando identificar relações entre ambas e possíveis contribuições clínicas no que diz respeito à compreensão da patologia, uma vez que a detecção de alterações visuais precoces pode auxiliar o processo diagnóstico.

Não foram encontrados estudos de revisão sistemática que avaliassem a evidência de prejuízo visual perceptivo em pacientes com TE. Desse modo, o objetivo deste trabalho consiste em fazer um levantamento geral sobre estudos com pacientes acometidos com TE, para preencher a lacuna encontrada na literatura quanto à interação percepção visual e TE para contribuir com a prática clínica, no que diz respeito ao processo diagnóstico e melhor prognóstico.

\section{MÉTODOS}

\section{Revisão da Literatura}

As diretrizes PRISMA (Liberati et al., 2009; Moher, Liberati, Tetzlaff, \& Altman, 2009) foram utilizadas para guiar esta revisão sistemática (número de registro PROSPERO: CRD42017054601). Uma busca eletrônica abrangente foi realizada nas bases de dados PubMed, BIREME, CINAHL, Web of Science, PsycINFO, SciELO, SCOPUS e ELSEVIER, desde a sua origem até 05 de janeiro de 2017. Os descritores específicos utilizados foram tremor, essential tremor, benign tremor, familial tremor, hereditary tremor e suas respectivas abreviações, juntamente com os termos visual processing, visual perception e vision. As palavras-chave foram escolhidas mesmo na ausência do termo específico (MESH), objetivando priorizar a sensibilidade sobre a especificidade do tema.

Durante a primeira triagem, dois revisores (DB ou TM) avaliaram os títulos e resumos de cada citação e excluíram os estudos que não se adaptavam aos critérios de elegibilidade destacados abaixo. Para cada estudo com potencial, dois revisores (NT ou TM) examinaram o artigo completo e avaliaram se os estudos encaixavam dentro dos critérios de inclusão. Quando em discordância, um terceiro avaliador foi contatado (RM).

\section{Seleção dos Estudos}

Dentre os critérios de inclusão destacam-se: (1) amostra clínica devidamente diagnosticada, de acordo com manuais diagnósticos reconhecidos; (2) ter avaliado a percepção visual; (3) estar em inglês, espanhol, francês ou português. Os critérios de exclusão foram: (1) amostra com animais e (2) cartas, editoriais e revisões de literatura.

Após exame dos artigos, de acordo com os critérios préestabelecidos, as seguintes variáveis foram isoladas com a finalidade de análise: (1) desenho do estudo, (2) instrumentos utilizados, (3) principais achados sobre percepção visual e (4) achados sobre outros domínios. Caso não existisse informação insuficiente nos estudos, como análise estatística ou resultados dos procedimentos, o respectivo autor seria contatado.

\section{Avaliação da Qualidade}

Cada estudo foi avaliado de acordo com o delineamento, a partir das diretrizes do National Institutes of Health (NIH). Os critérios de avaliação desta revisão são os mesmos preconizados pelas diretrizes do NIH. As diretrizes do NIH utilizadas foram estratificadas para estudos observacionais (14 critérios) e estudos de caso (nove critérios). Estes critérios englobam aspectos como (1) limitações (delineamento mal planejado, por exemplo); (2) consistência dos resultados; (3) precisão (capacidade de generalização dos achados e fornecimento de dados suficientes). Dois avaliadores preenchiam a classificação baseando-se em SIM ou NÃO para os critérios. Ao final, uma classificação geral foi atribuída para os estudos: Boa, Média ou Baixa evidência. 


\section{RESULTADOS}

A busca inicial nas bases de dados eletrônicas identificou 132 estudos potencialmente elegíveis. Após a triagem de citações por título e resumo, consideramos 12 artigos que foram lidos na íntegra. A Figura 1 demonstra o fluxograma de pesquisa desta revisão.

A maior parte dos artigos restringiu-se à estudos transversais, com tamanho da amostra variando de 1 (estudo de caso) a 183 pessoas por estudo. A Tabela 1 fornece as características principais dos estudos.

Esta análise baseia-se em 883 pacientes com TE (entre 21 e 81 anos), 746 controles e 217 com Doença de Parkinson (DdP). Em sua maioria, os estudos fizeram análise da percepção visual juntamente a funções cognitivas, como atenção, linguagem, aprendizagem e memória (Tabela 2 - material complementar). Os resultados indicaram que pessoas com TE apresentam baixa sensibilidade visual, apesar de não apresentarem prejuízos na visão de cores. $\mathrm{O}$ uso de grupo com DdP serviu de comparação e os resultados indicaram que quão mais severos os sintomas do transtorno de movimento, maior será a disfunção visual.

Estudos que avaliaram o rastreamento ocular indicaram a possibilidade de existência de (1) disfunção cerebelar e (2) supressão patológica do reflexo vestíbulo-ocular no TE, causando maior duração nos testes e alteração na latência do

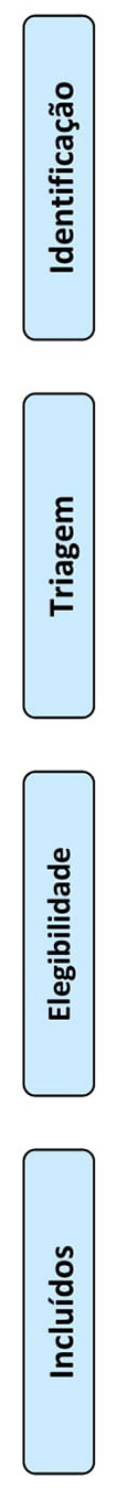
Registros identificados nas bases de dados $(n=132)$

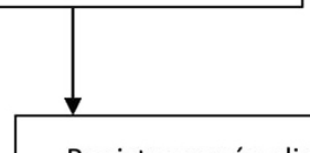

Registros após eliminação dos duplicados ( $n=91)$

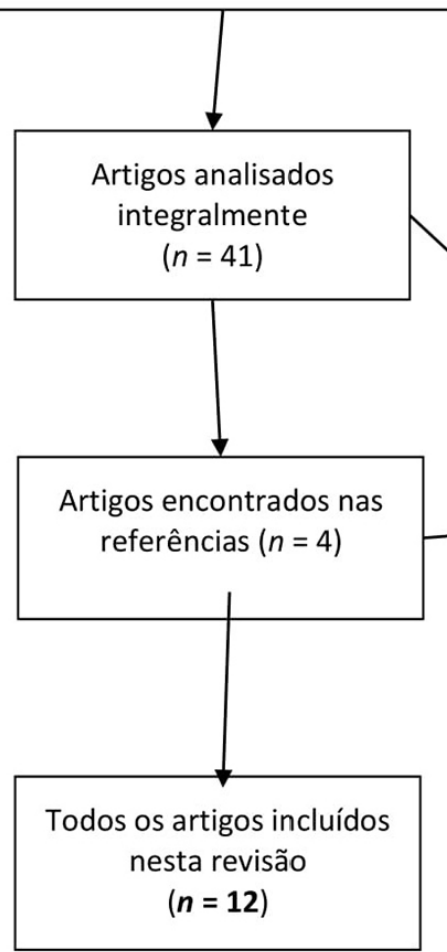

BIREME - 26

PubMed - 26

SCOPUS - 32

CINAHL -1

Web of Science -20

ELSEVIER - 27

PsycINFO - 0

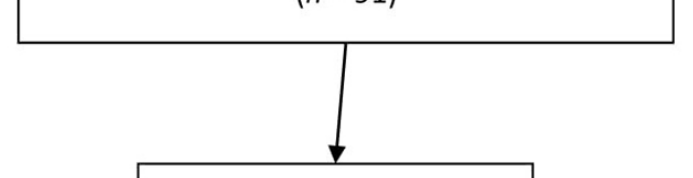


Tabela 1

Características dos estudos incluidos nesta revisão

\begin{tabular}{|c|c|c|c|c|c|}
\hline Estudo & Amostra & Objetivo & Instrumentos & $\begin{array}{l}\text { Domínios } \\
\text { Estudados }\end{array}$ & Principais achados \\
\hline $\begin{array}{l}\text { Benito- } \\
\text { León et al., } \\
2015\end{array}$ & $\begin{array}{l}23 \text { Pacientes com } \\
\text { TE e } 22 \text { controles } \\
\text { saudáveis; média de } \\
\text { idade }(D P) \text {, TE: } 63.3 \\
(13.4) \text {, controles: } \\
60.6(13.2) \text { anos }\end{array}$ & $\begin{array}{l}\text { Avaliar a relação } \\
\text { entre conectividade } \\
\text { anormal e severidade } \\
\text { do tremor essencial } \\
\text { nas funções visuais e } \\
\text { cognitivas }\end{array}$ & $\begin{array}{l}\text { Bateria de testes } \\
\text { neuropsicológicos e de } \\
\text { ressonância magnética } \\
\text { funcional }\end{array}$ & $\begin{array}{l}\text { Percepção } \\
\text { visual e funções } \\
\text { cognitivas }\end{array}$ & $\begin{array}{l}\text { Redução da percepção visual e } \\
\text { diminuição da conectividade funcional } \\
\text { das redes visuais }\end{array}$ \\
\hline $\begin{array}{l}\text { Bradvica et } \\
\text { al., } 2015\end{array}$ & $\begin{array}{l}51 \text { pacientes TE, } \\
59 \text { com doença } \\
\text { de Parkinson e } 54 \\
\text { indivíduos saudáveis } \\
\text { pareados em idade* }\end{array}$ & $\begin{array}{l}\text { Determinar a } \\
\text { especificidade dos } \\
\text { testes diagnósticos } \\
\text { para a disfunção de } \\
\text { cor e contraste visão } \\
\text { de contraste em } \\
\text { pacientes com TE } \\
\text { e DdP }\end{array}$ & $\begin{array}{l}\text { Cartela de Pelli- } \\
\text { Robson e placas } \\
\text { pseudoisocromáticas } \\
\text { de Ishihara (PPI) }\end{array}$ & $\begin{array}{l}\text { Sensibilidade } \\
\text { ao contraste e } \\
\text { visão de cores }\end{array}$ & $\begin{array}{l}\text { O grupo TE pertence ao grupo de } \\
\text { pessoas com sensibilidade ao contraste } \\
\text { baixa e o grupo DdP ao grupo de } \\
\text { pessoas com deficiência visual }\end{array}$ \\
\hline $\begin{array}{l}\text { Cubo et al., } \\
2010\end{array}$ & $\begin{array}{l}9 \text { pacientes com } \\
\text { DdP; } 8 \text { pacientes } \\
\text { com TE; } 9 \text { controles; } \\
\text { média de idade } \\
(D P), \text { TE: } 67.8(4.8) \text {, } \\
\text { DdP: } 64.1(12)\end{array}$ & $\begin{array}{l}\text { Medir a espessura } \\
\text { foveal em pacientes } \\
\text { com TE e compará- } \\
\text { la com população } \\
\text { normal e portadores } \\
\text { da DdP }\end{array}$ & $\begin{array}{l}\text { Tomografia de } \\
\text { coerência óptica }\end{array}$ & $\begin{array}{l}\text { Análise do } \\
\text { mapa de } \\
\text { espessura } \\
\text { macular e a } \\
\text { espessura do } \\
\text { centro da fóvea }\end{array}$ & $\begin{array}{l}\text { A espessura foveal média foi menor no } \\
\text { grupo DdP em comparação com o grupo } \\
\text { controle e TE, sendo mais fina no olho } \\
\text { contralateral ao lado mais afetado pelo } \\
\text { tremor }\end{array}$ \\
\hline $\begin{array}{l}\text { Gitchel et } \\
\text { al., } 2013\end{array}$ & $\begin{array}{l}60 \text { pacientes com } \\
\text { ET e } 60 \text { controles } \\
\text { pareados por } \\
\text { idade; média de } \\
\text { idade }(D P), \mathrm{TE} \\
\text { e controles: } 63.4 \\
(12.9) \text { anos }\end{array}$ & $\begin{array}{l}\text { Avaliar movimentos } \\
\text { oculares em pacientes } \\
\text { com TE }\end{array}$ & Eyelink II e TrakSTAR & $\begin{array}{l}\text { Rastreamento } \\
\text { do movimento } \\
\text { ocular }\end{array}$ & $\begin{array}{l}\text { Grupo TE apresentaram início de } \\
\text { movimento rápido e aceleração/ } \\
\text { desaceleração eficientes, como também } \\
\text { fixações oculares altamente estáveis }\end{array}$ \\
\hline $\begin{array}{l}\text { Helmchen } \\
\text { et al., } 2003\end{array}$ & $\begin{array}{l}17 \text { pacientes com } \\
\text { TE e } 11 \text { indivíduos } \\
\text { controle; média de } \\
\text { idade }(D P) \text {, TE: } 54.8 \\
(16.7) \text { e controles: } \\
56.6 \text { anos }\end{array}$ & $\begin{array}{l}\text { Observar relação } \\
\text { entre distúrbios do } \\
\text { movimento ocular e } \\
\text { disfunção cerebelar } \\
\text { em pacientes com TE }\end{array}$ & $\begin{array}{l}\text { Scleral search-coil e } \\
\text { eletro-oculografia }\end{array}$ & $\begin{array}{l}\text { Rastreamento } \\
\text { do movimento } \\
\text { ocular }\end{array}$ & $\begin{array}{l}\text { Movimento de perseguição suave } \\
\text { prejudicado, correlacionado com a } \\
\text { intensidade do tremor, e a supressão } \\
\text { patológica do reflexo vestíbulo-ocular }\end{array}$ \\
\hline $\begin{array}{l}\text { Jiménez- } \\
\text { Jiménez et } \\
\text { al., } 2010\end{array}$ & $\begin{array}{l}61 \text { pacientes com } \\
\text { TE e } 122 \text { controles } \\
\text { pareados por idade } \\
\text { e sexo; média de } \\
\text { idade }(D P), \text { TE: } 69.0 \\
(10.4), \text { DdP: } 68.5 \\
(7.4)\end{array}$ & $\begin{array}{l}\text { Investigar o padrão } \\
\text { de reação visual } \\
\text { e movimento de } \\
\text { pacientes com TE }\end{array}$ & $\begin{array}{l}\text { Bateria de testes } \\
\text { motores e visuais }\end{array}$ & $\begin{array}{l}\text { Tempo de } \\
\text { reação visual } \\
\text { e tempo de } \\
\text { movimento }\end{array}$ & $\begin{array}{l}\text { Grupo de paciente com TE apresentou } \\
\text { prejuízos significativos nos testes }\end{array}$ \\
\hline $\begin{array}{l}\text { Louis et } \\
\text { al., } 2012\end{array}$ & $\begin{array}{l}55 \text { casos TE e } 55 \\
\text { controles normais* }\end{array}$ & $\begin{array}{l}\text { Avaliar a existência } \\
\text { de anomalias na visão } \\
\text { de cores no TE }\end{array}$ & $\begin{array}{l}\text { Teste de } 100 \text { Matizes } \\
\text { de Farnsworth- } \\
\text { Munsell }\end{array}$ & Visão de cores & $\begin{array}{l}\text { Não foram detectados anormalidade de } \\
\text { visão de cores no grupo TE }\end{array}$ \\
\hline $\begin{array}{l}\text { Oh et al., } \\
2011\end{array}$ & $\begin{array}{l}54 \text { pacientes com } \\
\text { DdP, } 36 \text { pacientes } \\
\text { com TE e } 34 \\
\text { controles pareados } \\
\text { por idade; média de } \\
\text { idade }(D P) \text {, TE: } 66.3 \\
(6.8), \text { DdP: } 69.0 \\
(11.6), \text { Controles: } \\
65.4(9.5) \text { anos }\end{array}$ & $\begin{array}{l}\text { Comparar a disfunção } \\
\text { de discriminação de } \\
\text { cor entre os pacientes } \\
\text { com DdP e TE }\end{array}$ & $\begin{array}{l}\text { Teste de } 100 \text { Matizes } \\
\text { de Farnsworth- } \\
\text { Munsell, PPI }\end{array}$ & Visão de cores & $\begin{array}{l}\text { Não houve diferenças significativas na } \\
\text { discriminação de cores entre indivíduos } \\
\text { com TE }\end{array}$ \\
\hline $\begin{array}{l}\text { Piro et al., } \\
2016\end{array}$ & $\begin{array}{l}\text { Oito pacientes com } \\
\text { TE e oito pacientes } \\
\text { com DdP }\end{array}$ & $\begin{array}{l}\text { Avaliar o papel do } \\
\text { TE e DdP na visão de } \\
\text { cores }\end{array}$ & $\begin{array}{l}\text { PPI, Teste dicotômico } \\
\text { de Farnsworth }\end{array}$ & Visão de cores & $\begin{array}{l}66 \% \text { dos pacientes com TE } \\
\text { apresentaram Tritanopia. } 75 \% \text { dos } \\
\text { pacientes com DdP apresentaram } \\
\text { anomalias na visão de cores }\end{array}$ \\
\hline
\end{tabular}




\begin{tabular}{|c|c|c|c|c|c|}
\hline Estudo & Amostra & Objetivo & Instrumentos & $\begin{array}{l}\text { Domínios } \\
\text { Estudados }\end{array}$ & Principais achados \\
\hline $\begin{array}{l}\text { Piñero et } \\
\text { al., } 2015\end{array}$ & $\begin{array}{l}\text { Uma paciente de } 69 \\
\text { anos de idade }\end{array}$ & $\begin{array}{l}\text { Relatar alterações } \\
\text { na função de } \\
\text { sensibilidade ao } \\
\text { contraste (FSC) e } \\
\text { visão de cores }\end{array}$ & $\begin{array}{l}\text { Teste de } 100 \text { Matizes } \\
\text { de Farnsworth- } \\
\text { Munsell; Teste } \\
\text { CSV-1000E; Perímetro } \\
\text { multicanal }\end{array}$ & $\begin{array}{l}\text { Visão de cores, } \\
\text { sensibilidade ao } \\
\text { contraste }\end{array}$ & $\begin{array}{l}\text { A participante apresentou uma } \\
\text { diminuição da sensibilidade ao contraste } \\
\text { acromático em todas as frequências } \\
\text { espaciais em ambos os olhos (Teste } \\
\text { CSV-1000E). Nenhuma anormalidade } \\
\text { significativa de visão de cores foi } \\
\text { encontrado no teste Farnsworth-Munsell } \\
\text { 100. Os resultados do perímetro } \\
\text { multicanal confirmaram que houve } \\
\text { perdas significativas de sensibilidade } \\
\text { absoluta em comparação ao padrão } \\
\text { saudável no olho direito (OD) e uma } \\
\text { diminuição na sensibilidade do canal } \\
\text { azul-amarelo do olho esquerdo (OE) }\end{array}$ \\
\hline $\begin{array}{l}\text { Trillenberg } \\
\text { et al., } 2006\end{array}$ & $\begin{array}{l}12 \text { pacientes } \\
\text { ambulatoriais com } \\
\text { TE e } 14 \text { controles } \\
\text { pareados por idade. } \\
\text { Idade entre } 24 \text { a } 81 \\
\text { anos (média de } 55.6 \\
+/-16.5 \text { anos) }\end{array}$ & $\begin{array}{l}\text { Desmascarar qualquer } \\
\text { dismetria sacádica } \\
\text { cerebelar não } \\
\text { identificada }\end{array}$ & $\begin{array}{l}\text { Método da bobina } \\
\text { magnética; sonda de } \\
\text { emissores de ultrassom }\end{array}$ & $\begin{array}{l}\text { Rastreamento } \\
\text { ocular e } \\
\text { movimentos } \\
\text { das mãos }\end{array}$ & $\begin{array}{l}\text { As latências sacádicas do grupo TE não } \\
\text { foram significantemente reduzidas na } \\
\text { condição de olhar e apontar, ou seja, } \\
\text { resultados não diferem daquelas na } \\
\text { condição de apenas olhar. Amplitude } \\
\text { sacádica não diferiu entre os dois } \\
\text { grupos }\end{array}$ \\
\hline $\begin{array}{l}\text { Tröster et } \\
\text { al., } 1999\end{array}$ & $\begin{array}{l}40 \text { pacientes com } \\
\text { TE refratário, idade } \\
\text { média de } 72 \pm 8,5 \\
\text { anos. } 36 \text { fizeram } \\
\text { a cirurgia no lado } \\
\text { esquerdo e quatro no } \\
\text { lado direito }\end{array}$ & $\begin{array}{l}\text { Avaliar os efeitos } \\
\text { de curto prazo da } \\
\text { estimulação unilateral } \\
\text { talâmica profunda do } \\
\text { cérebro no TE }\end{array}$ & $\begin{array}{l}\text { Testes } \\
\text { neuropsicológicos }\end{array}$ & $\begin{array}{l}\text { Percepção } \\
\text { visual }\end{array}$ & $\begin{array}{l}\text { Não houve diferença significativa na } \\
\text { percepção visual e atenção visual }\end{array}$ \\
\hline
\end{tabular}

*não houve informações suficientes; DP: Desvio Padrão

DP: Desvio Padrão

início sacádico (Helmchen et al., 2003; Wójcik-Pędziwiatr et al., 2016). Observou-se também que a espessura foveal medida pela Tomografia de Coerência Óptica (OCT) pode ser um biomarcador promissor para TE e DdP (Satue et al., 2016; Sharifi et al., 2014).

Quando comparados com testes neurocognitivos, observou-se um maior tempo de resposta em tarefas de tempo de reação visual, habilidade visuomotora, tempo de movimento e marcha (Sahin et al., 2006; Sharifi at al., 2014). Os resultados demonstram que as capacidades normais visuomotoras podem coexistir com uma condição do tremor essencial (Schwartz, Badarny, Gofman, \& Hocherman, 1999). Além disso, um estudo que utilizou ressonância magnética funcional apresentou que o aumento da conectividade de redes de estado de descanso (redes frontoparietais) está associado a pior desempenho no processamento visual (Sharifi et al., 2014).

Estudos de neuroestimulação apresentaram resultados diferentes. Quando a estimulação foi do núcleo intermediário ventral do tálamo (VIM), o indivíduo não apresentou melhoras significativas nos testes cognitivos, mas observou melhora no controle do tremor, destreza manual e menor confusão, raiva, ansiedade e fadiga (Mandat, Koziara, Rola, Bonicki, \& Nauman, 2011; Zhang et al., 2010). Além disso, melhoras na memória, ansiedade e qualidade de vida foram observadas, mesmo após o período de 12 meses.

\section{DISCUSSÃO}

\section{Avaliação das Funções Visuais Básicas}

Sensibilidade ao contraste visual. A habilidade em detectar e interpretar detalhes de uma cena visual é determinada pela capacidade do sistema visual em distinguir padrões de contraste (Campbell \& Maffei, 1974). O contraste também pode ser entendido como a propriedade física do estímulo visual, sendo a magnitude da variação de luminância no estímulo relacionado com a luminância total de áreas adjacentes (Campbell \& Maffei, 1974; Shapley \& Enroth-Cugell, 1984). Isto é, mudanças na luminância do ambiente criam padrões de contraste que descrevem a maior parte da informação visual para o observador (Drew, 2005; Shapley \& Enroth-Cugell, 1984). 
O limiar de detecção do contraste é a quantidade mínima necessária para detectar corretamente um estímulo. Assim, sugere-se que o sistema visual humano (SVH) não codifica o padrão de contraste de uma vez, mas decompõe um padrão complexo de imagem em componentes bidimensionais, com canais que respondem de acordo com sua amplitude (DeValois \& DeValois, 1990). A relação funcional entre a recíproca do limiar de contraste e frequências espaciais é denominada função de sensibilidade ao contraste (FSC) (Cornsweet, 2012). A FSC está relacionada com diferenças existentes entre picos e vales de comprimentos de ondas em um espaço de coordenadas cartesianas ou polares, por exemplo. Neste sentido, o SVH possui alta sensibilidade quando um padrão precisa de pouco contraste para ser detectado. $\mathrm{O}$ inverso, a baixa sensibilidade, ocorre quando o SVH precisa de alto valor de contraste para detectar o estímulo. (DeValois \& DeValois, 1990; Santos \& Simas, 2001; Skottun, 2000). Uma vez que a sensibilidade ao contraste responde a frequências baixas (atribuída a aspectos neurais), médias e altas (atribuída a aspectos ópticos e neurais), a FSC é considerada uma rica descrição do processamento visual inicial (Wilson \& Humanski, 1993). Assim, a FSC fornece o liminar entre o visível e invisível (Pelli \& Bex, 2013).

Como uma extensão embrionária do tubo neural, a retina pode ser uma das regiões mais estudadas do sistema nervoso central devido a sua posição anatômica facilmente acessível (Adams \& Nasrallah, 2017). A retina permite uma investigação não invasiva do processamento visual. Nessa linha, vários tipos de medidas foram estabelecidos ao longo do tempo para caracterizar o processamento visual em indivíduos, como a acuidade visual e a FSC (Santos \& Simas, 2001). As representações visuais do mundo recebem interpretação ao serem processadas em áreas não-associativas do encéfalo. No entanto, em um nível de processamento inicial, o mundo toma forma para os indivíduos quando objetos (e estímulos) existentes no ambiente são decompostos e interpretados (Butler et al., 2005; Santos \& Simas, 2001). Medidas como a FSC permitem uma investigação detalhada das funções visuais por analisarem os aspectos ópticos e neurais, promovendo uma compreensão do processsamento visual inicial. A FSC pode ser utilizada para detectar alterações visuais em estágio inicial de transtornos neuropsiquiátricos e afecções neurológicas, compreender a problemática apresentada pelo paciente e colaborar para a manutenção desta condição. Por exemplo, o uso de certas medicações podem afetar o processamento visual, e reconhecer estas mudanças rapidamente pode promover um melhor prognóstico para os pacientes, a partir da mudança da medicação e, consequentemente, da melhor qualidade de vida (Bodis-Wollner, 1990a; Chen et al., 2003; Fernandes, Andrade, Andrade, Nogueira, \& Santos, 2017). A importância clínica de testes como a FSC é a de poder servir como uma rápida e fidedigna triagem para detecção de potenciais problemas visuais. Mensurações visuais podem servir como diagnósticos não invasivos do sistema visual humano (Fernandes, Almeida, \& Santos, 2017; Leat \& Woo, 1997; Moseley \& Hill, 1994).

Uma vez que a depleção dopaminérgica em pacientes com TE e DdP causa diminuição de dopamina na retina (principalmente nas células amácrinas e na camada plexiforme interna) e cerebelo, alguns estudos objetivaram utilizar a FSC como medida não invasiva que servisse de diagnóstico diferencial, quando comparado aos prejuízos motores (Bodis-Wollner, 1990; Bradvica, Bradvica, Matić, $\&$ Reisz-Majić, 2015; Piñero et al., 2015).

Como observado em outros estudos, a FSC diminui de acordo com a idade (Santos, Simas, \& Nogueira, 2003). A avaliação da FSC em pacientes com TE e DdP mais velhos apresenta um viés, uma vez que é esperado que os grupos de pacientes com mais de 50 anos apresentem uma redução na sensibilidade ao contraste. Todavia, o foco nestas condições é como a severidade do transtorno (em termos de funcionamento dopaminérgico) afetará a sensibilidade. De modo geral, pacientes com DdP apresentaram uma diminuição muito maior da SC em relação aos pacientes com $\mathrm{TE}$, os quais tiveram resultados similares ao grupo controle (Bradvica et al., 2015). A premissa abordada pelos estudos foi que o TE tem muito mais impacto no cerebelo do que na retina (Benito-León, 2008; 2014). No entanto, é importante citar que a maior parte dos casos de TE são diagnosticados por avaliação clínica que não faz menção à perturbações dopaminérgicas do sistema nervoso (Benito-León, 2014). Deste modo, o uso de medidas como DaTSCAN poderia ajudar na compreensão do funcionamento dopaminérgico no TE (Seifert \& Wiener, 2013).

Em contrapartida, um estudo de caso com uma paciente de 69 anos indicou redução na sensibilidade ao contraste, quando os olhos direito e esquerdo são comparados (Piñero et al., 2015). O uso do teste CSV-1000E permitiu observar existência de prejuízos nas vias magno-, parvo- e coniocelular. A diferença desse teste, em comparação aos procedimentos comuns de avaliação da visão periférica, reside no fato de que é um equipamento que permite a observações em cada uma das vias separadamente através de medidas com perímetro multicanal. $\mathrm{O}$ estudo de caso apontou a presença de um maior prejuízo no olho direito, em detrimento do esquerdo, ao comparar a população saudável. Todavia, apesar de mencionar que houve prejuízos em todas as frequências espaciais, o artigo só mostra uma frequência espacial ( 0.5 ciclos por grau de angulo visual), que, de acordo com a literatura, permitiria apenas avaliação detalhada do funcionamento da via magnocelular (DeValois \& DeValois, 1990; Skottun, 2015).

O uso de outros testes como o Teste de Contraste Multivision MCT 8000 Vistech (Vistech Consultants, Dayton, Ohio, EUA), ou Sistema de Teste de Visão de Contraste VCTS 6500 (Vistech Consultants), permitiria uma avaliação detalhada dos prejuízos na sensibilidade ao contraste nessa população. 
Controlar o efeito da idade em estudos com TE será um desafio. Todavia, comparar os resultados de indivíduos longitudinalmente ou com a própria população saudável permitirá dividir especificamente o papel da fisiopatologia do TE da idade.

$\mathrm{O}$ uso de medidas que avaliem as funções visuais básicas, principalmente a FSC, poderá servir como critério diagnóstico para sintomas não-motores, tornando, assim, mais fácil o diagnóstico diferencial.

\section{Avaliação Psicofísica da Visão de Cores}

Considerando os sintomas não-motores como características clínicas similares entre pacientes com TE e DdP, a visão de cores ainda é um domínio pouco estudado (Louis, Gerbin, \& Viner, 2012).

Os estudos que avaliaram a visão de cores no TE objetivaram adicionar ou remover evidências das similaridades entre TE e DdP (Bradvica et al., 2015; Louis et al., 2012; Oh et al., 2011; Piro et al., 2016). Isto é, caso existissem diferenças gradativas na visão de cores em $\mathrm{TE}$, provavelmente poderiam ter relação com a depleção dopaminérgica, que é característica principal da DdP (BodisWollner, 1990b).

No estudo realizado por Piro et al. (2016), foi utilizado o teste de 100 Matizes de Farnsworth-Munsell, teste dicotômico de Farnsworth ou as placas pseudoisocromáticas de Ishihara. Os resultados fornecidos por estes testes indicam a presença de prejuízos adquiridos ou congênitos da visão da cores. Ainda, três estudos avaliaram a discriminação de cores a partir do teste de 100 matizes de Farnsworth-Munsell com amostra e idade pareadas entre participantes (Louis et al., 2012; Oh et al., 2011; Piñero et al., 2015). Os resultados não mostraram diferenças significativas entre os participantes com TE, apesar da diminuição da sensibilidade a cores.

É imprescindível mencionar que não há um consenso na literatura acerca de valores-base para considerar que $o$ indivíduo apresenta perda na visão de cores azul-amarelo ou verde-vermelho. Todavia, o total de erros (Total Error Score [TES]) encontrados nos estudos (TES entre 115 e 140) já podem indicar perdas visuais se compararmos a outros estudos com populações saudáveis (Cranwell, Pearce, Loveridge, \& Hurlbert, 2015; Kinnear \& Sahraie, 2002)

\section{Avaliação do Rastreamento do Movimento Ocular}

Através das avaliações psicofísicas, pesquisadores desenvolveram testes de avaliação da percepção visual através de imagens acromáticas e acromáticas. Todavia, técnicas eletrofisiológicas e de rastreamento do movimento ocular são baseadas em novas tecnologias que também permitem uma descrição apurada da apresentação do estímulo, registro, sincronização e análise (Santos et al., 2014).
Dados clínicos (Benito-León et al., 2015) indicam que o cerebelo está envolvido na fisiopatologia do TE. Deste modo, a avaliação dos movimentos oculares permite observar se há relação entre distúrbios do movimento ocular e disfunção cerebelar neste grupo de pacientes.

A maior parte dos estudos apontou movimento de perseguição suave prejudicado, correlacionado com a intensidade do tremor e supressão patológica do reflexo vestíbulo-ocular (Gitche et al., 2013; Helmchen et al., 2003; Jiménez-Jiménez et al., 2010; Trillenberg et al., 2006). Além disso, as latências sacádicas do grupo TE não foram significantemente reduzidas na condição de olhar e apontar, ou seja, os resultados não diferem daquelas na condição de apenas olhar.

Novamente, estes resultados apontam para um maior papel do cerebelo em detrimento da depleção dopaminérgica. Todavia, utilizar apenas o rastreamento do movimento ocular não examina como a percepção da forma interage com o funcionamento cortical (Bühren, Terzi, Bach, Wesemann, $\&$ Kohnen, 2006; Cornsweet, 2012; Drew, 2005). Com isso, uma medida adicional, como a tomografia de coerência óptica (Optical Coherence Tomography [OCT]), pode servir como indicador de alterações retinianas.

\section{Avaliação Óptica no Tremor Essencial}

Recentemente, medidas de avaliação como a OCT têm permitido investigar mudanças estruturais da retina em diversas condições (Koster et al., 2002; Sharifi et al., 2014). A vantagem desse método é que, se existem alterações estruturais na retina, o sinal chegará ampliado ou reduzido ao córtex visual primário. Deste modo, alterações estruturais e funcionais serviriam como biomarcadores de transtornos como DdP (devido à diminuição dopaminérgica e afinamento das camadas retinianas) e do TE.

De modo geral, a OCT cria uma imagem de alta resolução e em três dimensões da retina, provendo uma janela em tempo real das mudanças estruturais que podem ser associadas a processos neurodegenerativas, como no TE (Boeke, Rosen, Mastrianni, Xie, \& Bernard, 2016).

Estudos que utilizaram a avaliação óptica no TE encontraram redução da espessura da camada de fibras nervosas retinianas (Turkel et al., 2015). Esses resultados indicam que as mudanças podem servir como critérios diagnósticos para sintomas não-motores e podem dar suporte ao fato de que o TE pode ser um transtorno neurodegenerativo, como a DdP (Boeke et al., 2016).

\section{Uma Visão Geral}

Esta revisão indicou que a percepção visual é prejudicada no TE, afetando a maior parte dos pacientes (Tabela 1). Ainda, a severidade do tremor afeta diretamente as habilidades visuomotoras nos pacientes (Schwartz et al., 1999). 
Os resultados demonstram que as capacidades normais visuomotoras podem coexistir com uma condição do TE, indicando que os mecanismos neuronais que produzem tremor não são distintos daqueles que suportam coordenação visuomotora (Jiménez-Jiménez et al., 2010). As hipóteses relacionadas à neurobiologia do TE apontam para disfunções dopaminérgicas (envolvendo vias de transmissão) ou alterações estruturais, como no cerebelo, formando o cerne da compreensão fisiológica do TE. Igualmente, a informação visual que chega ao córtex perpassa caminhos subjacentes ao TE, como cerebelo e tálamo, por exemplo (Piñero et al., 2015; Sharifi et al., 2014).

A presença de fatores biosociodemográficos, como gênero ou nível de educação gerando efeitos na severidade do TE, não foram relatados (Tabela 1). Os estudos não mostraram consistência ao estratificar e analisar os subgrupos das amostras.

A severidade do prejuízo na percepção visual em indivíduos com TE parece estar associada ao funcionamento difuso do lobo parietal e occipital direito (Sharifi et al., 2014). Também observou-se correlação com radiações talâmica anterior, fascículos longitudinais e uncinados inferiores, além do lobo posterior do cerebelo.

Algumas limitações precisam ser mencionadas, tanto dos estudos selecionados quanto do processo de revisão. Primeiramente, observou-se uma grande heterogeneidade metodológica entre os estudos, trazendo, assim, baixa consistência interna dentre eles. Apesar desta revisão não se tratar de uma meta-análise, sabe-se que grandes diferenças metodológicas impedem que façamos uma perfeita síntese quantitativa dos estudos. Apesar do grande número de participantes nos estudos, o motivo dos diferentes resultados pode ser atribuído às diferenças metodológicas.

Talvez seja cedo para afirmar, mas os prejuízos visuais no TE podem formar as principais características clínicas não-motoras, uma vez que a visão pode ser uma das portas para a cognição (Pylyshyn, 1999). Obviamente, faz-se necessário uso de baterias de testes neuropsicológicos para corroborar esta premissa.

Do outro lado, os diferentes tamanhos das amostram trouxeram resultados diferentes (Bradvica et al., 2015; Oh et al., 2011; Piñero et al., 2015; Piro et al., 2016). Estudos com amostra maior apresentaram similaridade ou melhor desempenho dos indivíduos com TE (Jiménez-Jiménez et al., 2010; Louis et al., 2012; Oh et al., 2011). Em contrapartida, estudos com amostra menor indicaram o contrário (Piñero et al., 2015; Piro et al., 2016). Por este motivo, a generalização destes estudos deve ser feita com bastante cautela, uma vez que parece haver a necessidade de metodologias mais rigorosas.

Apesar da baixa força de evidência dos estudos e da heterogeneidade dos resultados, esta é a primeira revisão sistemática a traçar paralelos sobre a percepção visual e o tremor essencial. Deste modo, observamos que estes são imprescindíveis para continuidade de mais estudos.

Prevendo que o número de estudos seria baixo, o nosso objetivo inicial não foi executar metanálise e técnicas de meta-regressão. Em vez disso, uma revisão crítica das principais conclusões sobre a investigação do TE e percepção visual foi realizada, dirigindo as suas limitações.

\section{CONCLUSÕES}

Apesar da heterogeneidade dos dados, observamos que existem prejuízos visuais em pessoas com TE e estes podem servir como biomarcadores não-motores. Sugere-se a realização de estudos com metodologias mais rigorosas que avaliem os aspectos ópticos e neurais da visão, provendo, assim, resultados menos diferentes. Os potenciais benefícios desta abordagem são o desenvolvimento de medidas fidedignas que gerem programas de intervenção e prevenção antecipadas.

\section{REFERÊNCIAS}

Adams, S. A., \& Nasrallah, H. A. (2017). Multiple retinal anomalies in schizophrenia. Schizophrenia Research. https://doi. org/10.1016/j.schres.2017.07.018

Benito-León, J. (2008). Essential tremor: from a monosymptomatic disorder to a more complex entity. Neuroepidemiology, 31(3), 191-192. https://doi.org/10.1159/000154933

Benito-León, J. (2014). Essential tremor: A neurodegenerative disease? Tremor and Other Hyperkinetic Movements, 4(252), 1-9. https://doi.org/10.7916/D8765CG0

Benito-León, J., Louis, E. D., Romero, J. P., Hernández-Tamames, J. A., Manzanedo, E., Álvarez-Linera, J., ... Rocon, E. (2015). Altered functional connectivity in essential tremor: A resting-state fMRI study. Medicine, 94(49), e1936. https://doi. org/10.1097/MD.0000000000001936
Bodis-Wollner, I. (1990). Visual deficits related to dopamine deficiency in experimental animals and Parkinson's disease patients. Trends in Neurosciences, 13(7), 296-302.

Boeke, A., Rosen, D., Mastrianni, J., Xie, T., \& Bernard, J. (2016). Optical coherence tomography as potential biomarker in Parkinson's disease and Alzheimer's disease (P5.177). Neurology, 86(16 Supplement), P5.177.

Bradvica, I. S., Bradvica, M., Matić, S., \& Reisz-Majić, P. (2015). Visual dysfunction in patients with Parkinson's disease and essential tremor. Neurological Sciences, 36(2), 257-262. https://doi.org/10.1007/s10072-014-1930-2

Bühren, J., Terzi, E., Bach, M., Wesemann, W., \& Kohnen, T. (2006). Measuring contrast sensitivity under different lighting conditions: comparison of three tests. Optometry and Vision 
Science: Official Publication of the American Academy of Optometry, 83(5), 290-298. https://doi.org/10.1097/01. opx.0000216100.93302.2d

Butler, P. D., Zemon, V., Schechter, I., Saperstein, A. M., Hoptman, M. J., Lim, K. O., ... Javitt, D. C. (2005). Early-stage visual processing and cortical amplification deficits in schizophrenia. Archives of General Psychiatry, 62(5), 495-504. https://doi. org/10.1001/archpsyc.62.5.495

Campbell, F. W., \& Maffei, L. (1974). Contrast and spatial frequency. Scientific American, 231(5), 106-114. https://doi. org/10.1038/scientificamerican1174-106

Chen, Y., Levy, D. L., Sheremata, S., Nakayama, K., Matthysse, S., \& Holzman, P. S. (2003). Effects of typical, atypical, and no antipsychotic drugs on visual contrast detection in schizophrenia. The American Journal of Psychiatry, 160(10), 1795-1801. https://doi.org/10.1176/appi.ajp.160.10.1795

Cornsweet, T. N. (2012). Visual perception. Massachusetts: Academic Press.

Cranwell, M. B., Pearce, B., Loveridge, C., \& Hurlbert, A. C. (2015). Performance on the Farnsworth-Munsell 100-Hue Test is significantly related to Nonverbal IQFM100 Test significantly related to NVIQ. Investigative Ophthalmology \& Visual Science, 56(5), 3171-3178. https://doi.org/10.1167/iovs.14-16094

Deuschl, G., Wenzelburger, R., Löffler, K., Raethjen, J., \& Stolze, H. (2000). Essential tremor and cerebellar dysfunction clinical and kinematic analysis of intention tremor. Brain: A Journal of Neurology, 123(Pt 8), 1568-1580.

DeValois, R. L., \& DeValois, K. K. (1990). Spatial Vision. Oxford University Press.

Drew, S. (2005). Anatomy And physiology of the eye, contrast, contrast sensitivity, luminance perception and psychophysics. Recuperado de https://www.ict.griffith.edu.au > docs > humanvision

Fernandes, T. M. P., Almeida, N. L. de, \& Santos, N. A. dos. (2017) Effects of smoking and smoking abstinence on spatial vision in chronic heavy smokers. Scientific Reports, 7(1), 1690. https:// doi.org/10.1038/s41598-017-01877-z

Fernandes, T. M. P., Andrade, S. M., Andrade, M. J. O., Nogueira, R. M. T. B. L., \& Santos, N. A. (2017). Colour discrimination thresholds in type 1 Bipolar Disorder: a pilot study. Scientific Reports, 7(1), 16405. https://doi.org/10.1038/s41598-01716752-0

Filip, P., Lungu, O. V., Manto, M.-U., \& Bareš, M. (2016). Linking essential tremor to the cerebellum: Physiological evidence. Cerebellum (London, England), 15(6), 774-780. https://doi. org/10.1007/s12311-015-0740-2

Gazzaniga, M. S. (2004). The cognitive neurosciences. Massachusetts: MIT Press.

Gitchel, G. T., Wetzel, P. A., \& Baron, M. S. (2013). Slowed saccades and increased square wave jerks in essential tremor. Tremor and Other Hyperkinetic Movements 3(3). https://doi. org/10.7916/D8251GXN

Helmchen, C., Hagenow, A., Miesner, J., Sprenger, A., Rambold, H., Wenzelburger, R., ... Deuschl, G. (2003). Eye movement abnormalities in essential tremor may indicate cerebellar dysfunction. Brain: A Journal of Neurology, 126(Pt 6), 1319-1332.

Jiménez-Jiménez, F. J., Rubio, L., Alonso-Navarro, H., Calleja, M., Pilo-de-la-Fuente, B., Plaza-Nieto, J. F., ... Agúndez, J. A. G. (2010). Impairment of rapid repetitive finger movements and visual reaction time in patients with essential tremor. European Journal of Neurology, 17(1), 152-159. https://doi. org/10.1111/j.1468-1331.2009.02784.x

Kinnear, P. R., \& Sahraie, A. (2002). New Farnsworth-Munsell 100 hue test norms of normal observers for each year of age 5-22 and for age decades 30-70. British Journal of Ophthalmology, 86(12), 1408-1411. https://doi.org/10.1136/bjo.86.12.1408
Koller, W. C., Busenbark, K., \& Miner, K. (1994). The relationship of essential tremor to other movement disorders: Report on 678 patients. Annals of Neurology, 35(6), 717-723. https:// doi.org/10.1002/ana.410350613

Koster, B., Deuschl, G., Lauk, M., Timmer, J., Guschlbauer, B., \& Lucking, C. (2002). Essential tremor and cerebellar dysfunction: abnormal ballistic movements. Journal of Neurology, Neurosurgery, and Psychiatry, 73(4), 400-405. https://doi.org/10.1136/jnnp.73.4.400

Leat, S. J., \& Woo, G. C. (1997). The validity of current clinical tests of contrast sensitivity and their ability to predict reading speed in low vision. Eye (London, England), 11(Pt 6), 893-899. https://doi.org/10.1038/eye.1997.228

Liberati, A., Altman, D. G., Tetzlaff, J., Mulrow, C., Gøtzsche, P. C., Ioannidis, J. P. A., ... Moher, D. (2009). The PRISMA statement for reporting systematic reviews and meta-analyses of studies that evaluate healthcare interventions: explanation and elaboration. BMJ, 339, b2700. https://doi.org/10.1136/ bmj.b2700

Louis, E. D., Gerbin, M., \& Viner, A. S. (2012). Color Vision: A study of essential tremor cases vs. normal controls. European Journal of Neurology, 19(8), 1136-1139. https://doi. org/10.1111/j.1468-1331.2012.03688.x

Mandat, T., Koziara, H., Rola, R., Bonicki, W., \& Nauman, P. (2011). Thalamic deep brain stimulation in the treatment of essential tremor. Neurologia I Neurochirurgia Polska, 45(1), 37-41.

Marsden, J. F., Ashby, P., Limousin-Dowsey, P., Rothwell, J. C., \& Brown, P. (2000). Coherence between cerebellar thalamus, cortex and muscle in man. Brain, 123(7), 1459-1470. https:// doi.org/10.1093/brain/123.7.1459

Moher, D., Liberati, A., Tetzlaff, J., \& Altman, D. G. (2009). Preferred reporting items for systematic reviews and metaanalyses: The PRISMA statement. BMJ, 339, b2535. https:// doi.org/10.1136/bmj.b2535

Moseley, M. J., \& Hill, A. R. (1994). Contrast sensitivity testing in clinical practice. The British Journal of Ophthalmology, 78(10), 795-797.

Oh, Y. S., Kim, J. S., Chung, S. W., Song, I. U., Kim, Y. D., Kim, Y. I., \& Lee, K.-S. (2011). Color vision in Parkinson's disease and essential tremor. European Journal of Neurology, 18(4), 577-583. https://doi.org/10.1111/j.1468-1331.2010.03206.x

Pelli, D. G., \& Bex, P. (2013). Measuring contrast sensitivity. Vision Research, 90, 10-14. https://doi.org/10.1016/j. visres.2013.04.015

Piñero, D. P., Monllor, B., Moncho, V., Camps, V. J., \& de Fez, D. (2015). Visual function alterations in essential tremor: A case report. Journal of Innovative Optical Health Sciences, 08(05), 1550040-1550045. https://doi.org/10.1142/ S1793545815500406

Piro, A., Tagarelli, A., Nicoletti, G., Lupo, A., Fletcher, R., \& Quattrone, A. (2016). Parkinson's disease, Parkinsonism, de novo Parkinson's disease, essential tremor: A color vision preliminary analysis. Parkinsonism \& Related Disorders, 22, e42-e43. https://doi.org/10.1016/j.parkreldis.2015.10.070

Pylyshyn, Z. (1999). Is vision continuous with cognition? The case for cognitive impenetrability of visual perception. The Behavioral and Brain Sciences, 22(3), 341-423.

Sahin, H. A., Terzi, M., Uçak, S., Yapici, O., Basoglu, T., \& Onar, M. (2006). Frontal functions in young patients with essential tremor: A case comparison study. The Journal of Neuropsychiatry and Clinical Neurosciences, 18(1), 64-72. https://doi.org/10.1176/jnp.18.1.64

Santos, N. A. dos, Neto, C., Arruda, A. de, Sousa, B. M. de, Pessoa, E. D. C., \& Nogueira, R. M. T. B. L. (2014). Matlab e rastreamento ocular: Aplicações em psicofísica e processos psicológicos básicos. Temas em Psicologia, 22(3), 589-601. https://doi.org/10.9788/TP2014.3-05 
Santos, N. A. dos, \& Simas, M. L. de B. (2001). Contrast sensitivity function: Indicator of the visual perception of form and of the spatial resolution. Psicologia: Reflexão e Crítica, 14(3), 589-597. https://doi.org/10.1590/S0102-79722001000300014

Santos, N. A. dos, Simas, M. L. de B., \& Nogueira, R. M. T. B. L. (2003). Visual processing of form in the aged: Threshold contrast curves to angular and spatial frequency. Psicologia: Reflexão e Crítica, 16(2), 271-277. https://doi.org/10.1590/ S0102-79722003000200007

Satue, M., Obis, J., Rodrigo, M. J., Otin, S., Fuertes, M. I., Vilades, E., ... Garcia-Martin, E. (2016). Optical coherence tomography as a biomarker for diagnosis, progression, and prognosis of neurodegenerative diseases. Journal of Ophthalmology, 2016, e8503859. https://doi.org/10.1155/2016/8503859

Schönfeldt-Lecuona, C., Kregel, T., Schmidt, A., Pinkhardt, E. H., Lauda, F., Kassubek, J., ... Gahr, M. (2016). From imaging the brain to imaging the retina: Optical Coherence Tomography (OCT) in schizophrenia. Schizophrenia Bulletin, 42(1), 9-14. https://doi.org/10.1093/schbul/sbv073

Schwartz, M., Badarny, S., Gofman, S., \& Hocherman, S. (1999). Visuomotor performance in patients with essential tremor. Movement Disorders: Official Journal of the Movement Disorder Society, 14(6), 988-993.

Seifert, K. D., \& Wiener, J. I. (2013). The impact of DaTscan on the diagnosis and management of movement disorders: A retrospective study. American Journal of Neurodegenerative Disease, 2(1), 29-34.

Shapley, R., \& Enroth-Cugell, C. (1984). Chapter 9 Visual adaptation and retinal gain controls. Progress in Retinal Research, 3(C), 263-346. https://doi.org/10.1016/0278-4327(84)90011-7

Sharifi, S., Nederveen, A. J., Booij, J., \& van Rootselaar, A.F. (2014). Neuroimaging essentials in essential tremor: A systematic review. NeuroImage : Clinical, 5, 217-231. https:// doi.org/10.1016/j.nicl.2014.05.003
Skottun, B. C. (2000). The magnocellular deficit theory of dyslexia: The evidence from contrast sensitivity. Vision Research, 40(1), 111-127. https://doi.org/10.1016/S00426989(99)00170-4

Skottun, B. C. (2015). On the use of spatial frequency to isolate contributions from the magnocellular and parvocellular systems and the dorsal and ventral cortical streams. Neuroscience and Biobehavioral Reviews, 56, 266-275. https://doi.org/10.1016/j. neubiorev.2015.07.002

Trillenberg, P., Führer, J., Sprenger, A., Hagenow, A., Kömpf, D., Wenzelburger, R., ... Helmchen, C. (2006). Eye-hand coordination in essential tremor. Movement Disorders, 21(3), 373-379. https://doi.org/10.1002/mds.20729

Tröster, A. I., Fields, J. A., Pahwa, R., Wilkinson, S. B., Straits-Tröster, K. A., Lyons, K., ... Koller, W. C. (1999). Neuropsychological and quality of life outcome after thalamic stimulation for essential tremor. Neurology, 53(8), 1774-1774. https://doi.org/10.1212/WNL.53.8.1774

Turkel, Y., Ornek, N., Dag, E., Ornek, K., Alpua, M., Ogurel, T., \& Olmez, Y. (2015). Retinal nerve fiber layer thickness in patients with essential tremor. Neurology Asia, 20(20), 363-366.

Wilson, H. R., \& Humanski, R. (1993). Spatial frequency adaptation and contrast gain control. Vision Research, 33(8), 1133-1149. https://doi.org/10.1016/0042-6989(93)90248-U

Wójcik-Pędziwiatr, M., Plinta, K., Krzak-Kubica, A., Zajdel, K., Falkiewicz, M., Dylak, J., ... Rudzińska, M. (2016). Eye movement abnormalities in essential tremor. Journal of Human Kinetics, 52(1), 53-64. https://doi.org/10.1515/ hukin-2015-0193

Zhang, K., Bhatia, S., Oh, M. Y., Cohen, D., Angle, C., \& Whiting, D. (2010). Long-term results of thalamic deep brain stimulation for essential tremor. Journal of Neurosurgery, 112(6), 1271-1276. https://doi.org/10.3171/2009.10. JNS09371 